and Parker, M.J. 2008. Mortality following hip fracture: trends and geographical variations over the last 40 years. Injury. 39:1157-1163.

4. Lyles, K.W., et al. 2007. Zoledronic acid and clinical fractures and mortality after hip fracture. $N$. Engl. J. Med. 357:1799-1809.

5. Canalis, E., Giustina, A., and Bilezikian, J.P. 2007. Mechanisms of anabolic therapies for osteoporosis. N. Engl. J. Med. 357:905-916.

6. Krishnan, V., Bryant, H.U., and Macdougald, O.A 2006. Regulation of bone mass by Wnt signaling. J. Clin. Invest. 116:1202-1209.

7. Macsai, C.E., Foster, B.K., and Xian, C.J. 2008. Roles of Wnt signalling in bone growth, remodelling, skeletal disorders and fracture repair. J. Cell. Physiol. 215:578-587

8. Kuhl, M., and Pandur, P. 2008. Dorsal axis duplication as a functional readout for Wnt activity. Methods Mol. Biol. 469:467-476.

9. Hurlstone, A.F., et al. 2003. The Wnt/beta-catenin pathway regulates cardiac valve formation. Nature. 425:633-637

10. Korinek, V., et al. 1998. Depletion of epithelial stem-cell compartments in the small intestine of mice lacking Tcf-4. Nat. Genet. 19:379-383.
11. Logan, C.Y., and Nusse, R. 2004. The Wnt signaling pathway in development and disease. Annu. Rev. Cell Dev. Biol. 20:781-810.

12. Nusse, R., et al. 1991. A new nomenclature for int -1 and related genes: the Wnt gene family. Cell. 64:231.

13. Sharma, R.P., and Chopra, V.L. 1976. Effect of the Wingless (wg1) mutation on wing and haltere development in Drosophila melanogaster. Dev. Biol. 48:461-465.

14. Nusse, R., and Varmus, H.E. 1982. Many tumors induced by the mouse mammary tumor virus contain a provirus integrated in the same region of the host genome. Cell. 31:99-109.

15. Brown, A.M., Papkoff, J., Fung, Y.K., Shackleford, G.M., and Varmus, H.E. 1987. Identification of protein products encoded by the proto-oncogene int-1. Mol. Cell. Biol. 7:3971-3977.

16. Brown, A.M., Wildin, R.S., Prendergast, T.J., and Varmus, H.E. 1986. A retrovirus vector expressing the putative mammary oncogene int- 1 causes partial transformation of a mammary epithelial cell line. Cell. 46:1001-1009.

17. Kansara, M., et al. 2009. Wnt inhibitory factor 1 is epigenetically silenced in human osteosarcoma, and targeted disruption accelerates osteosarcomagenesis in mice. J. Clin. Invest. 119:837-851.

18. Baylin, S.B., Herman, J.G., Graff, J.R., Vertino, P.M., and Issa, J.-P. 1998. Alterations in DNA methylation: a fundamental aspect of neoplasia. Adv. Cancer Res. 72:141-196.

19. Suzuki, H., et al. 2004. Epigenetic inactivation of SFRP genes allows constitutive WNT signaling in colorectal cancer. Nat. Genet. 36:417-422.

20. Herman, J.G., et al. 1995. Inactivation of the CDKN2/p16/MTS1 gene is frequently associated with aberrant DNA methylation in all common human cancers. Cancer Res. 55:4525-4530.

21. Liggett, W.H., Jr., and Sidransky, D. 1998. Role of the p16 tumor suppressor gene in cancer. J. Clin. Oncol. 16:1197-1206.

22. van de Wetering, M., et al. 2002. The beta-catenin/ TCF-4 complex imposes a crypt progenitor phenotype on colorectal cancer cells. Cell. 111:241-250.

23. Adams, P.D., and Enders, G.H. 2008. Wnt signaling and senescence: A tug of war in early neoplasia? Cancer Biol. Ther. 7:1706-1711.

24. Gnant, M., et al. 2009. Endocrine Therapy plus Zoledronic Acid in Premenopausal Breast Cancer. N. Engl.J. Med. 360:679-691.

\title{
Can licorice lick colon cancer?
}

\author{
Paul M. Stewart ${ }^{1}$ and Stephen M. Prescott ${ }^{2}$
}

\begin{abstract}
${ }^{1}$ College of Medical and Dental Sciences, University of Birmingham, Birmingham, United Kingdom. ${ }^{20 k l a h o m a ~ M e d i c a l ~ R e s e a r c h ~ F o u n d a t i o n, ~}$
\end{abstract} Oklahoma City, Oklahoma, USA.

\begin{abstract}
COX-2 promotes colon cancer. While both nonselective NSAIDs and selective COX-2 inhibitors reduce disease burden, their adverse gastrointestinal and cardiovascular side effects limit their therapeutic use. In this issue of the JCI, Zhang et al. used gene silencing and a derivative of licorice root to show that inhibition of the enzyme $11 \beta$-hydroxysteroid dehydrogenase type II (11ßHSD2) reduces tumor COX-2 activity, tumor growth, and metastasis by increasing the tonic glucocorticoid-mediated suppression of the COX-2 signaling pathway without the adverse effects associated with NSAIDs and selective COX-2 inhibitors (see the related article beginning on page 876). Their findings suggest that 11 $\beta$ HSD2 inhibition may be a potential therapeutic option in colon cancer, warranting further investigation.
\end{abstract}

COX-2 is a crucial enzyme in the synthesis of prostaglandins and prostacyclin, which play a variety of roles in the regulation of cell growth, hemostasis, sensing of pain, and inflammation. In normal colon tissue, there is little or no expression of COX-2; however, COX-2 expression is induced early in colon carcinogenesis, is key to disease progression, and influences the clinical course of disease (Figure 1, A and $\mathrm{B}$, and reviewed in ref. 1 ). The COX-2

Conflict of interest: The authors have declared that no conflict of interest exists.

Nonstandard abbreviations used: GE, glycyrrhetinic acid; $11 \beta$ HSD, $11 \beta$-hydroxysteroid dehydrogenase; MR mineralocorticoid receptor.

Citation for this article: J. Clin. Invest. 119:760-763

(2009). doi:10.1172/JCI38936. response clearly plays a central role in colon carcinogenesis, because inhibitors of COX-2 enzymatic activity prevent the development of intestinal polyps in mice and humans (reviewed in ref. 1), and deletion of Cox2 in mice almost completely protects the animals from the development of these polyps (reviewed in ref. 1). However, enthusiasm for the prevention of colon cancer via pharmacological COX-2 inhibition has been tempered by the recognition that such a prevention strategy inherently requires long-term exposure to COX-2 inhibitors. Unfortunately, traditional NSAIDs, which are nonselective COX inhibitors, can cause gastrointestinal hemorrhage, among other complications (2), while selective COX-2 inhibitors confer an increased risk of cardiovascular death (3). Thus, a detailed understanding of how COX-2 expression is induced would be potentially valuable from two perspectives - it would provide insight into both the molecular steps involved in carcinogenesis and potential therapeutic targets.

That COX-2 is overexpressed in colon polyps and cancer has been recognized for more than 15 years (reviewed in ref. 1), but the molecular basis for this overexpression has remained unclear despite extensive investigation of the regulation of the COX2 gene in many experimental settings. It is likely that what was originally thought to be a cell-autonomous event is instead a response to extracellular signals - a "field effect," with growth factors providing much of the signal that results in induction of COX2. From the time of the discovery of COX2 as an early inducible gene, it was almost immediately recognized that COX2 induction in vitro could be inhibited by a class of steroid hormones known as glucocorticoids $(4,5)$. This pharmacologic effect has been attributed to changes in both COX-2 transcription and mRNA stability (6). However, it was not known whether COX-2 was regulated by endogenous glucocorticoids, the most important of which 


\section{A Resting colon cell}

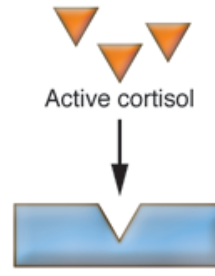

GC receptor

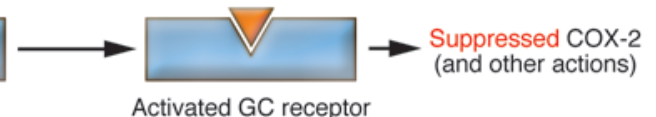

B Colon cell overexpressing $11 \beta \mathrm{HSD} 2$

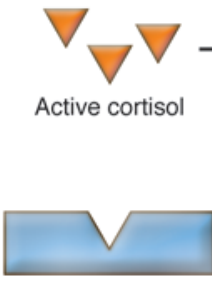

GC receptor
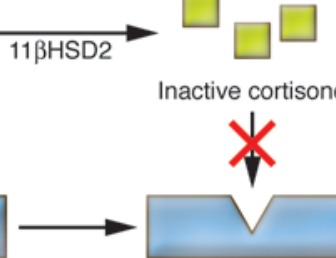

Inactive $\mathrm{GC}$ receptor

C Colon cell overexpressing $11 \beta \mathrm{HSD} 2$ and treated with "licorice"

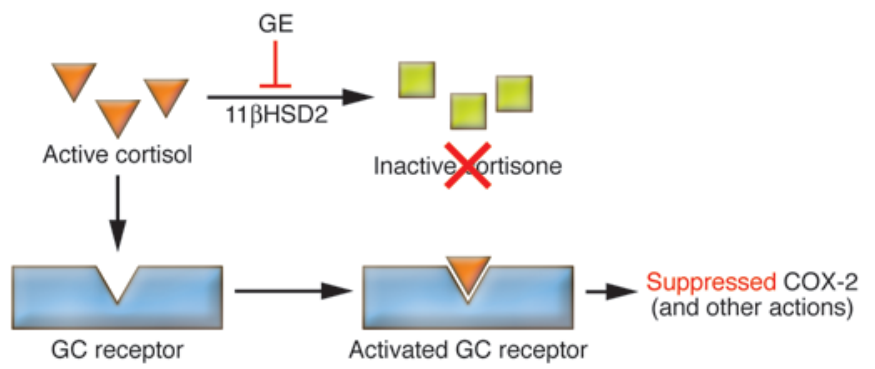

is cortisol in humans, as it supports a variety of important metabolic, cardiovascular, immunologic, and homeostatic functions.

\section{The actions of cortisol are regulated in target tissues}

Endogenous cortisol secretion is regulated by the hypothalamo-pituitary-adrenal axis, which largely dictates the levels of circulating glucocorticoids and tissue exposure. However, within target tissues, the exposure of cortisol to corticosteroid receptors is also regulated through the activity of steroid metabolism pathways, notably via the expression of $11 \beta$-hydroxysteroid dehydrogenases (11ßHSDs). Two isoforms of $11 \beta$ HSD exist: the type I oxoreductase, $11 \beta$ HSD1, which can generate active cortisol from the inactive keto-form, cortisone; and the type II $11 \beta$ HSD 2 isoform, a highly efficient NAD-dependent dehydrogenase responsible for the reverse reaction, converting active cortisol to inactive cortisone (Figure 1B and ref. 7).

Because $11 \beta$ HSD 1 is expressed in glucocorticoid receptor-rich tissues such as liver, adipose tissue, and muscle, there is fervent interest in its therapeutic inhibition in patients with metabolic syndrome. The rationale for this interest is that inhibition of the local generation of cortisol in liver and omental fat reduces hepatic gluconeogenesis and glucose output and reduces omental adipogenesis and lipolysis, thereby reducing the waist/hip ratio and lowering levels of circulating lipids (8).

In contrast, in adult tissues, $11 \beta \mathrm{HSD} 2$ is expressed in epithelial cells in mineralocorticoid receptor-rich (MR-rich) tissues such as kidney, colon, and salivary gland. Here it acts in an autocrine fashion to protect the MR - which, paradoxically, in vitro has the same inherent affinity for the mineralocorticoid aldosterone as it does for the glucocorticoid cortisol - from illicit occupancy by cortisol (9).

Expression of 11ßHSD2 has also been reported in cancers, most notably in endocrine tumors such as pituitary and adrenal adenomas $(10,11)$, but also in osteosarcoma, renal, breast, and lung cancer cells (12). The underlying explanation for aberrant

\section{Figure 1}

Inhibition of $11 \beta \mathrm{HSD} 2$ blocks COX-2 and suppresses colon carcinogenesis. (A) In resting colon cells, COX-2 expression is suppressed by the binding of endogenous cortisol to the glucocorticoid (GC) receptor. (B) In the current study, Zhang et al. show that the expression of $11 \beta \mathrm{HSD} 2$ is increased both in human colon adenomas and in intestinal adenomas in $\mathrm{Apc}^{+/ \mathrm{min}}$ mice (14). Active cortisol is converted by $11 \beta \mathrm{HSD} 2$ to inactive cortisone that is unable to activate the glucocorticoid receptor. This releases the repression of COX-2, which is then expressed at high levels and generates signals (primarily prostaglandins) that promote colon tumorigenesis. (C) The authors also show that these cellular events could be reversed - at least with regard to this signaling pathway - by inhibiting the enzymatic activity of $11 \beta \mathrm{HSD} 2$ via gene silencing or pharmacologically with the licorice root derivative GE. Under these conditions, cortisol is available to suppress COX-2 expression via the glucocorticoid receptor and therefore suppress tumorigenesis.
$11 \beta$ HSD2 expression is uncertain, but it has been postulated to control glucocorticoid regulation of cellular proliferation (reviewed in ref. 13). Results from in vitro studies using malignant transformed cell lines demonstrate the antiproliferative actions of glucocorticoids; thus, the local inactivation of cortisol by $11 \beta \mathrm{HSD} 2$ may be an important oncogenic process promoting cellular proliferation. In vitro, $11 \beta \mathrm{HSD} 1$ brings about changes opposite to those mediated by $11 \beta \mathrm{HSD} 2$ - the local generation of cortisol suppresses cellular proliferation (13). Arguably, for this reason, there are very few malignant transformed cell lines that express the $11 \beta \mathrm{HSD} 1$ isoform.

\section{Inhibition of 11ßHSD2 blocks COX-2 activity and tumor growth}

In this issue of the JCI, Zhang et al. demonstrate similar findings in the colon (14). They report that $11 \beta \mathrm{HSD} 2 \mathrm{mRNA}$ and the immunoreactive $11 \beta$ HSD2 protein itself are overexpressed in both human colon adenomas and in intestinal adenomas in $A \mathrm{Ac}^{+/ \min }$ mice - which are heterozygous for a 
nonsense mutation in the Apc gene, homologous to human germline and somatic APC mutations, and consequently develop intestinal adenomas - and that this overexpression correlates with increased COX-2 expression and activity (Figure 1B). They demonstrate that gene silencing or pharmacological inhibition of $11 \beta$ HSD 2 with the licorice root extract glycyrrhetinic acid (GE) reduced tumor COX-2 activity, tumor growth, and metastasis via inhibition of the COX-2-mediated signaling pathway (Figure 1C). Encouragingly, the authors did not observe any increase in atherogenesis or decrease in the measure of systemic prostacyclin levels after $11 \beta \mathrm{HSD} 2$ inhibition. Their findings suggest that $11 \beta$ HSD2 inhibition prevents colon cancer by selectively blocking tumor COX-2 activity and that this can be achieved without triggering adverse side effects in the cardiovascular system that are associated with selective COX-2 inhibitors (3).

Zhang et al. (14) suggest that all or most of the carcinogenic actions of $11 \beta \mathrm{HSD} 2$ occur via the COX-2 pathway, including via cytosolic phospholipase $\mathrm{A}_{2}\left(\mathrm{cPLA}_{2}\right)$ and microsomal prostaglandin $\mathrm{E}$ synthase (mPGES-1), the initial and terminal enzymes, respectively, in COX-2-mediated prostaglandin synthesis. It is not clear whether increased expression of COX-2, and subsequent signaling through prostaglandin $\mathrm{E}_{2}$, is sufficient to cause cancer - it may depend on the tissue and other stimuli. For example, the answer to this question with respect to mammary tissue seems to be a qualified yes, since transgenic mice with tissue-specific overexpression of COX-2 develop tumors without an additional known inducer, but only after multiple pregnancies (15). In contrast, forced expression of COX-2 in skin sensitizes mice to cancer but is insufficient to trigger disease alone (16). Similar observations have been made in mice with intestine-specific COX-2 expression (17). Thus, if the majority of the effects of $11 \beta$ HSD 2 are mediated via the COX-2 pathway, we predict that COX-2 overexpression will not initiate cancer, but will promote it.

\section{Is licorice - or its relatives - a candy or a therapeutic?}

Should we now regard $11 \beta$ HSD 2 as a therapeutic target in patients with colon cancer? Evidence dating back to ancient Eastern civilizations suggests that there is something to this story. Several naturally occurring compounds are known to inhibit $11 \beta \mathrm{HSD} 2$, including GE and flavanoids such as naringenin, found in grapefruit juice. Many of these are ingredients of Eastern herbal medicines such as shakuyaku-kanzo-to and oren-gedoku-to, which have been shown in clinical studies to treat a variety of tumors, including hepatomas and colon cancers (18). Some in vitro studies have also demonstrated an effect via COX-2 activity (19).

However, there are two broad issues to consider. The first is whether one would expect inhibition of $11 \beta$ HSD2 to be as good as, or superior to, inhibition of COX-2; the second is whether long-term exposure to such a class of drugs would be safe. With respect to the first issue, there is reason for optimism because there is a good theoretical basis for blocking expression of COX-2 as a therapeutic strategy. Lack of COX-2mediated prostaglandin $\mathrm{E}_{2}$ production in tumors would block adenoma formation, tumor growth, and angiogenesis, returning the status of the tissue to that of normal colon tissue. In addition, there is another theoretical benefit: NSAIDs and COX-2 inhibitors can themselves induce the expression of COX-2 under some circumstances $(20,21)$, which could lead to a paradoxical increase in prostaglandin synthesis if COX-2 were induced and then the enzymatic inhibition was relieved. Such relief of inhibition could occur if a patient was not perfectly compliant with the dosage schedule of the inhibitor, which is common during long-term therapy. With respect to the issue of long-term safety, more evidence is needed of the effects of $11 \beta$ HSD 2 inhibition, and the outcomes to date of the use of selective COX-2 inhibitors are sobering (3). Zhang et al. recognize this concern and provide encouraging data that $11 \beta \mathrm{HSD} 2$ inhibition did not show systemic suppression of prostacyclin levels and that there was no worsening of disease in a mouse model of atherosclerosis (14).

The 11ßHSD2 inhibitor used in the current report (14) was glycyrrhizic acid, which the authors showed was converted to the active metabolite GE. The GE content of licorice differs across the globe - it is high in Asia and some European countries, such as Holland, but undetectable in confectionary licorice sold in the United States, where tobacco sticks and gums contain the highest quantities of GE.

At this time, tissue-specific 11ßHSD2 inhibition is not available, and while GE might have beneficial effects on colon carcinoma, it will also inhibit renal 11ßHSD2 expression and activity (22). Despite the reassurances of Zhang et al. as to the lack of cardiovascular side effects of $11 \beta$ HSD2 inhibition compared with COX-2 inhibition (14), this is unlikely to be the case. Because the MR cannot differentiate between the mineralocorticoid, salt-retaining properties of cortisol or aldosterone, and plasma cortisol concentrations are 1,000 -fold greater than aldosterone concentrations, even the most trivial inhibition or lack of renal $11 \beta$ HSD2 will result in salt-dependent mineralocorticoid hypertension, the speed of onset and severity of which would be directly related to the degree of $11 \beta$ HSD2 inhibition (23). However, if the present findings are confirmed, locally acting enteric $11 \beta$ HSD 2 inhibitors that are not systemically absorbed may be a way forward in colon cancer therapeutics.

Address correspondence to: Paul. M. Stewart, College of Medical and Dental Sciences, University of Birmingham, Room 236, Institute of Biomedical Research, Wolfson Drive, Edgbaston, Birmingham B15 2TT, United Kingdom. Phone: 44-121415-8708; Fax; 44-121-415-8712; E-mail: p.m.stewart@bham.ac.uk. Or to: Stephen M. Prescott, Oklahoma Medical Research Foundation, 825 NE 13th Street, Oklahoma City, Oklahoma 73104, USA. Phone: (405) 271-7210; Fax: (405) 227-5809; E-mail: steve-prescott@omrf.org.

1. Prescott, S.M., and Fitzpatrick, F.A. 2000. Cyclooxygenase-2 and carcinogenesis. Biochim. Biophys. Acta. 1470:M69-M78.

2. Thun, M.J., Henley, S.J., and Patrono, C. 2002. Nonsteroidal anti-inflammatory drugs as anticancer agents: mechanistic, pharmacological, and clinical issues. J. Natl. Cancer Inst. 94:252-266.

3. Grosser, T., Fries, S., and FitzGerald, G.A. 2006. Biological basis for the cardiovascular consequences of COX-2 inhibition: therapeutic challenges and opportunities. J. Clin. Invest. 116:4-15.

4. Kujubu, D.A., and Herschman, H.R. 1992. Dexamethasone inhibits mitogen induction of the TIS10 prostaglandin synthase/cyclooxygenase gene. J. Biol. Chem. 267:7991-7994.

5. Lee, S.H., et al. 1992. Selective expression of mitogen-inducible cyclooxygenase in macrophages stimulated with lipopolysaccharide. J. Biol. Chem. 267:25934-25938.

6. Newton, R., Seybold, J., Kuitert, L.M.E., Bergmann, M., and Barnes, P.J. 1998. Repression of cyclooxygenase- 2 and prostaglandin $\mathrm{E}_{2}$ release by transcriptional and post-transcriptional mechanisms involving loss of polyadenylated mRNA. J. Biol. Chem. 273:32312-32321.

7. Tomlinson, J.W., et al. 2004. 11beta-hydroxysteroid dehydrogenase type 1: a tissue specific regulator of glucocorticoid response. Endocr. Rev. 25:831-866.

8. Tomlinson, J.W., and Stewart, P.M. 2005. Selective inhibition of 11beta hydroxysteroid dehydrogenase type 1 as a novel treatment for the metabolic syndrome. Nat. Clin. Pract. Endocrinol. Metab. 1:92-99.

9. Edwards, C.R.W., et al. 1988. Localisation of 11 
beta-hydroxysteroid dehydrogenase - tissue specific protector of the mineralocorticoid receptor. Lancet. 8618:986-989.

10. Korbonits, M., et al. 2001. Expression of 11beta hydroxysteroid dehydrogenase isozymes in the human pituitary: induction of the type 2 enzyme in corticotropinomas and other pituitary tumours. J. Clin. Endocrinol. Metab. 86:2728-2733.

11. Mune, T., et al. 2003. Role of local 11beta hydroxysteroid dehydrogenase type 2 expression in determining the phenotype of adrenal adenomas. J. Clin. Endocrinol. Metab. 88:864-870.

12. Sasano, H., et al. 1997. Localization of mineralocorticoid receptor and 11 beta hydroxysteroid dehydrogenase type II in human breast and its disorders. Anticancer Res. 17:2001-2007.

13. Rabbitt, E.H., et al. 2002. Pre-receptor regulation of glucocorticoid action by 11 beta hydroxysteroid dehydrogenase: a novel determinant of cell proliferation. FASEB J. 16:36-44.
14. Zhang, M.-Z., et al. 2009. Inhibition of $11 \beta$-hydroxysteroid dehydrogenase type II selectively blocks the tumor COX-2 pathway and suppresses colon carcinogenesis in mice and humans. J. Clin. Invest. 119:876-885

15. Liu, C.H., et al. 2001. Overexpression of cyclooxygenase- 2 is sufficient to induce tumorigenesis in transgenic mice. J. Biol. Chem. 276:18563-18569.

16. Muller-Decker, K., et al. 2002. Transgenic cyclooxygenase- 2 overexpression sensitizes mouse skin for carcinogenesis. Proc. Natl. Acad. Sci. U. S. A. 99:12483-12488.

17. Al-Salihi, M.A., et al. 2008. Transgenic expression of cyclooxygenase- 2 in mouse intestine epithelium is insufficient to initiate tumorigenesis but promotes tumor progression. Cancer Lett. 273:225-232.

18. Hibasami, H., Iwase, H., Yoshioka, K., and Takahashi, H. 2006. Glycyrrhetinic acid (a metabolic substance and aglycon of glycyrrhizin) induces apoptosis in human hepatoma, promyelotic leukemia and stomach cancer cells. Int. J. Mol Med. 17:215-219.

19. Fukutake, M., et al. 2000. Suppressive effect of the herbal medicine Oren-gedoku-to on cyclooxygenase 2 activity and azoxymethane-induced aberrant crypt foci development in rats. Cancer Lett. 157:9-14.

20. Meade, E.A., McIntyre, T.M., Zimmerman, G.A., and Prescott, S.M. 1999. Peroxisome proliferators enhance cyclooxygenase-2 expression in epithelial cells. J. Biol. Chem. 274:8328-8334.

21. Pang, L., Nie, M., Corbett, L., and Know, A.J. 2003. Cyclooxygenase- 2 expression by nonsteroidal antiinflammatory drugs in human airway smooth muscle cells: role of peroxisome proliferator-activated receptors. J. Immunol. 170:1043-1051.

22. Stewart, P.M., et al. 1987. Mineralocorticoid activity of liquorice:11beta hydroxysteroid dehydrogenase deficiency comes of age. Lancet. 8563:821-824.

23. Quinkler, M., and Stewart, P.M. 2003. Hypertension and the cortisol cortisone shuttle. J. Clin. Endocrinol. Metab. 88:2384-2392.

\title{
The voltage-gated $\mathrm{K}^{+}$channel subunit Kv1.1 links kidney and brain
}

\author{
David H. Ellison ${ }^{1,2}$ \\ 1Division of Nephrology \& Hypertension, Department of Medicine, Department of Physiology \& Pharmacology, and Heart Research Center, \\ Oregon Health \& Science University, Portland, Oregon, USA. ${ }^{2 V A}$ Medical Center, Portland, Oregon, USA.
}

\begin{abstract}
Analysis of Mendelian $\mathrm{Mg}^{2+}$ wasting disorders helps us to unravel the mechanisms of $\mathrm{Mg}^{2+}$ homeostasis. In this issue of the JCI, Glaudemans and colleagues show that mutations in voltage-gated $\mathrm{K}^{+}$channel subtype 1.1 (Kv1.1) cause autosomal dominant hypomagnesemia in humans (see the related article beginning on page 936). Interestingly, other mutations in the same protein cause the neurological disease episodic ataxia type 1 . The authors show, using cells with heterologous expression of the wild-type and mutant channels, that the mutant channel is dysfunctional and speculate that $\mathrm{Mg}^{2+}$ wasting results from changes in apical membrane voltage along the nephron. Mechanisms by which the apical voltage is generated and how Kv1.1 fits within this context are discussed herein.
\end{abstract}

Rare Mendelian diseases are windows into both physiology and pathogenesis. Examples include the rare $\mathrm{Mg}^{2+}$ wasting disorders that form the basis for most of our current understanding of renal $\mathrm{Mg}^{2+}$ transport. Several proteins that mediate $\mathrm{Mg}^{2+}$ transport, both around and through cells, have now been identified and cloned, using positional cloning approaches. Sec-

Conflict of interest: The author has declared that no conflict of interest exists.

Nonstandard abbreviations used: CNT, connecting tubule; DCT; distal convoluted tubule; ENaC, epithelial $\mathrm{Na}^{+}$channel; KCNA1, $\mathrm{K}^{+}$voltage-gated channel, Shaker-related subfamily, member $1 ;$ Kv1.1, voltagegated $\mathrm{K}^{+}$channel subtype 1.1 ; ROMK, renal outer medullary $\mathrm{K}^{+}$channel; TAL, thick ascending limb; TRPM6, transient receptor potential cation channel, subfamily M, member 6 .

Citation for this article: J. Clin. Invest. 119:763-766 (2009). doi:10.1172/JCI38835. ondary dysfunction of these proteins may also contribute to hypomagnesemia in the critically ill, where the incidence has been estimated as $20 \%-60 \%$ and has been associated with excess mortality (1). Hypomagnesemia is often drug related, with diuretics, calcineurin inhibitors, and antineoplastic agents (e.g., cisplatin and cetuximab) common offenders (2). The study of Mendelian disorders of $\mathrm{Mg}^{2+}$ homeostasis has also led to the identification of novel and sometimes unexpected regulatory pathways that impact transport pathways secondarily.

Eighty percent of plasma $\mathrm{Mg}^{2+}$ is ultrafilterable by glomeruli. Whereas the majority of every other ion studied to date is reabsorbed along the proximal tubule, proximal $\mathrm{Mg}^{2+}$ reabsorption constitutes only $10 \%-15 \%$ of the filtered load. In contrast, the thick ascending limb (TAL) reabsorbs approximately $70 \%$ of filtered $\mathrm{Mg}^{2+}$ and clearly plays a central role in regulating $\mathrm{Mg}^{2+}$ excretion. What surprised many investigators, however, was that most disorders of $\mathrm{Mg}^{2+}$ balance result from dysfunction along the distal convoluted tubule (DCT), a short nephron segment that, just a few years ago, was believed to play only a minor role in $\mathrm{Mg}^{2+}$ homeostasis (3). The DCT is now recognized as important not only for $\mathrm{Mg}^{2+}$ balance, but also for the control of $\mathrm{Na}^{+}, \mathrm{K}^{+}$, and $\mathrm{Ca}^{2+}$ levels (4). In this issue of the JCI, Glaudemans and colleagues report that missense mutations in $\mathrm{K}^{+}$voltage-gated channel, Shaker-related subfamily, member 1 (KCNA1), which encodes voltage-gated $\mathrm{K}^{+}$channel subtype 1.1 (Kv1.1) expressed by DCT cells, causes autosomal dominant hypomagnesemia in humans (5). Surprisingly, other mutations in the same gene cause episodic ataxia type 1 (EA1) (6), a neurological syndrome in which hypomagnesemia has not been reported. In the present study, the investigators showed that Kv1.1 localizes to the apical membrane of DCT cells, where the transient receptor potential cation channel, subfamily M, member 6 (TRPM6) controls $\mathrm{Mg}^{2+}$ entry, driven by its electrochemical potential. Expression studies showed that the mutated Kv1.1 protein, while having no direct effect on TRPM6, exhibited reduced 